\title{
Facilitation of learning spatial relations among locations by visual cues: Implications for theoretical accounts of spatial learning
}

\author{
BRADLEY R. STURZ \\ Armstrong Atlantic State University, Savannah, Georgia \\ Michael F. BROWN \\ Villanova University, Villanova, Pennsylvania \\ AND \\ DEBbIE M. Kelly \\ University of Saskatchewan, Saskatoon, Saskatchewan, Canada
}

\begin{abstract}
Human participants searched in a real environment or interactive 3-D virtual environment open field for four hidden goal locations arranged in a $2 \times 2$ square configuration in a $5 \times 5$ matrix of raised bins. The participants were randomly assigned to one of two groups: cues + pattern or pattern only. The participants experienced a training phase, followed by a testing phase. Visual cues specified the goal locations during training only for the cues + pattern group. Both groups were then tested in the absence of visual cues. The results in both environments indicated that the participants learned the spatial relations among goal locations. However, visual cues during training facilitated learning of the spatial relations among goal locations: In both environments, the participants trained with the visual cues made fewer errors during testing than did those trained only with the pattern. The results suggest that learning based on the spatial relations among locations may not be susceptible to cue competition effects and have implications for standard associative and dual-system accounts of spatial learning.
\end{abstract}

Mobile animals appear to rely on at least two sources of spatial information: landmarks and environmental geometry (for a review, see Shettleworth, 1998). The use of landmarks involves determining location and orientation by using objects in the environment (Gallistel, 1990). In contrast, the use of environmental geometry involves determining location and orientation by using the geometric properties of the surrounding enclosure (for reviews, see Cheng \& Newcombe, 2005, 2006).

Although both sources of spatial information permit learning of location, they differ with respect to how location is referenced. Spatial learning based on landmarks permits the learning of location with reference to discrete visual landmarks, and a wide range of species have been shown to use discrete visual landmarks in order to locate a hidden goal (for a review, see Healy, 1998). In contrast, spatial learning based on environmental geometry permits the learning of location without reference to discrete visual landmarks but, instead, with reference to the geometric properties of the surrounding enclosure, and various vertebrate species have been shown to use the overall geometry of the surrounding experimental environment in order to locate a hidden goal (for reviews, see Cheng \& Newcombe, 2005, 2006).
Much debate remains as to whether these sources of spatial information are learned collectively by a unitary associative-based system (e.g., Chamizo, 2003; Graham, Good, McGregor, \& Pearce, 2006; Miller \& Shettleworth, 2007; Pearce, Graham, Good, Jones, \& McGregor, 2006) or independently by separate feature- and geometry-based systems (e.g., Cheng, 1986; Cheng \& Newcombe, 2006; Gallistel, 1990). In addition, a new account of geometrybased learning has been proposed in terms of the encoding of distances and directions from boundaries (Doeller \& Burgess, 2008; Doeller, King, \& Burgess, 2008; for a review, see Burgess, 2006). This dual-system model proposes that one system is dedicated to learning distance and direction from boundaries, whereas the other is dedicated to learning distance and direction from landmarks.

An important part of the empirical evidence that has been used to discriminate unitary- and dual-system models of spatial learning is associative cue competition (e.g., overshadowing and/or blocking). Existence of competition between spatial cues has been taken as evidence that they are processed by the same learning system, whereas the absence of competition has been taken as evidence that they are processed by separate learning systems (e.g., Chamizo, 2003; Cheng, 2008; Shettleworth, 1998).

B. R. Sturz, bradley.sturz@armstrong.edu 
The dual-system models predict an immunity of either geometry or boundary learning to cue competition from landmarks, but both dual-system models and standard associative accounts predict cue competition among landmarks (see Burgess, 2006; Chamizo, 2003; Cheng, 1986; Gallistel, 1990; however, see Cheng, Shettleworth, Huttenlocher, \& Rieser, 2007).

In the present experiment, we tested for cue competition in a search task in which boundaries and environmental geometry were rendered irrelevant for the purpose of informing theories of spatial learning. Specifically, the experimental design allowed us to examine whether learning based on visual landmarks would overshadow learning based on the spatial relations among hidden goal locations. The design followed that of Brown and Terrinoni (1996), who introduced a search task in which rats learned the spatial relations among hidden goal locations. Critically, the location of the goals was unpredictable across trials in relation to landmarks and environmental geometry. Thus, only the spatial relations among the goal locations were predictive. Brown, Yang, and DiGian (2002) used the same search task with rats but manipulated whether visual landmarks, in addition to the spatial relations among the goal locations, were available as cues. They found no evidence of cue competition (overshadowing) between the landmarks and spatial relations among locations, which suggests that there may be spatial cues other than environmental geometry or distance to boundaries that are immune to cue competition from landmarks.

In the present experiment, one group of human participants was trained in the presence of visual cues that marked goal locations, and another group was trained in their absence. Goal locations maintained a consistent spatial relationship with each other but varied across trials with respect to boundaries and environmental geometry. Both groups were then tested in the absence of visual cues in order to determine their effect on learning the spatial relations among goal locations. According to a unitarysystem model, the group of participants trained with the visual cues should learn less about the spatial relations among locations because of the presence of these cues and, as a result, their performance should be inferior to that of the group trained in the absence of visual cues. Although dual-system models predict the absence of cue competition in the presence of environmental geometry or boundaries, neither geometry nor boundaries can be used to determine goal locations in the present task. Accordingly, like a unitary-system model, dual-system models predict that participants trained with the visual cues should learn less about the spatial relations among locations, as compared with participants trained in the absence of these cues.

Logistical problems associated with testing humans in navigational tasks have resulted in recent research utilizing 3-D virtual environments (for a review, see Kelly \& Gibson, 2007). Despite extensive use of virtual environments in spatial research, relatively few direct comparisons have been made between human navigation in real and virtual environments (see Klatzky, Loomis, Beall, Chance, \& Golledge, 1998; Sturz, Bodily, Katz, \& Kelly,
2009). As a result, real and virtual versions of the search task were used in parallel in order to allow explicit comparisons of the mechanisms used by humans to navigate real and virtual environments.

\section{METHOD}

\section{Real Environment}

\section{Participants}

Forty University of Saskatchewan undergraduate students (20 male and 20 female) served as participants. The participants received extra class credit.

\section{Apparatus and Stimuli}

A search space $(5.55 \mathrm{~m}$ in length $\times 3.74 \mathrm{~m}$ in width $\times 5.30 \mathrm{~m}$ in height) was created by hanging white opaque curtains from ceiling to floor of an experimental room. The floor was covered with shredded paper. Twenty-five raised bins $(22 \mathrm{~cm}$ in diameter $\times 18.5 \mathrm{~cm}$ in height), which also contained shredded paper, were arranged in a $5 \times 5$ matrix within the room (see Figure 1, top panels).

\section{Procedure}

The participants were randomly assigned to one of two groups: cues + pattern or pattern only. Each group consisted of 20 participants (10 male and 10 female). The participants completed two phases: training and testing. Each phase consisted of 15 trials in which four small, red plastic balls were hidden under shredded paper in four bins arranged in a $2 \times 2$ square pattern within the $5 \times 5$ bin matrix. The four goal locations always formed a square pattern (i.e., balls always maintained the same spatial relationship to each other) but varied in an otherwise unpredictable manner across trials. The participants searched for these four balls. The participants began each trial at position S (Figure 1). A choice was defined as when a participant fully inserted his or her hand into a bin. When all four balls had been retrieved, the participants returned the balls to the experimenter and exited the open field. Otherwise, the participants continued searching until all the balls had been retrieved.

Training. Training consisted of 15 trials. For each trial, the square pattern was randomly assigned to one of 16 possible configurations of locations. Four bins were thereby designated as goal locations, and one ball was placed under shredded paper within each of these goal locations. Training was identical for the cues + pattern and pattern-only groups, with the exception that goal locations (bins) were marked in green for the cues + pattern group. The remaining bins were unmarked (terra cotta colored).

Testing. Testing consisted of 15 trials. For each trial, the square pattern was randomly assigned to one of the 16 locations. The four bins were then designated as goal locations, and one ball was placed within each of these goal locations. All the bins were unmarked, and testing was identical for the cues + pattern and pattern-only groups.

\section{Virtual Environment}

\section{Participants}

A combination of 40 ( 7 from Armstrong Atlantic State University, 33 from Villanova University) undergraduate students (20 male and 20 female) served as participants. They received extra credit or participated as a part of a course requirement.

\section{Apparatus}

An interactive 3-D virtual environment was constructed and rendered using Valve Hammer Editor and was run on the Half-Life Team Fortress Classic platform. A personal computer, a 19-in. flatscreen LCD monitor, an optical mouse, a keyboard, and headphones served as the interface with the virtual environment. The monitor $(1,152 \times 864$ pixels $)$ provided a first-person perspective of the virtual environment (see Figure 1, middle panels). The arrow keys 


\section{Cues + Pattern Training}
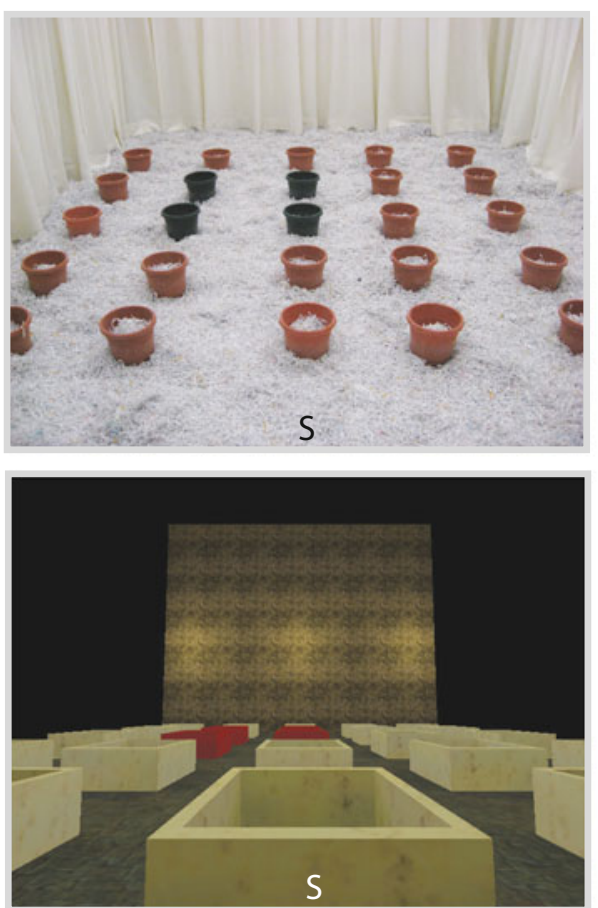

Group
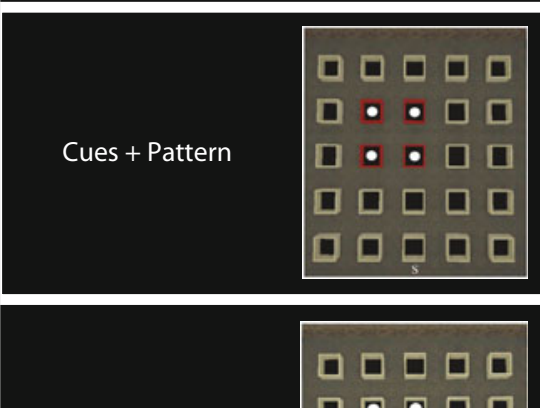

Pattern Only

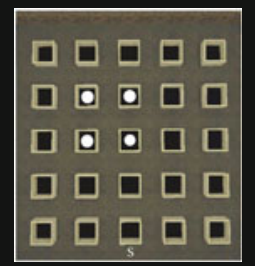

Pattern-Only Training
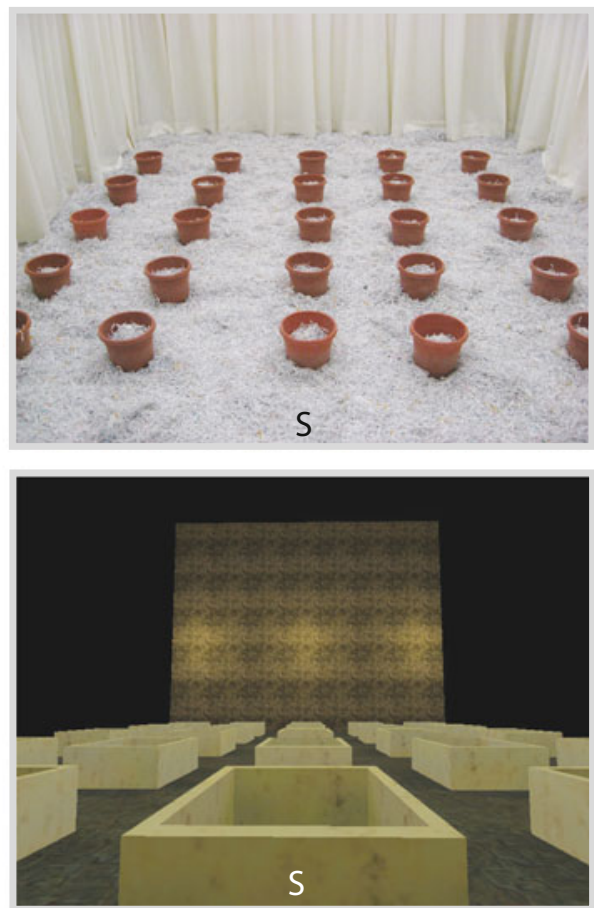

Training

Testing
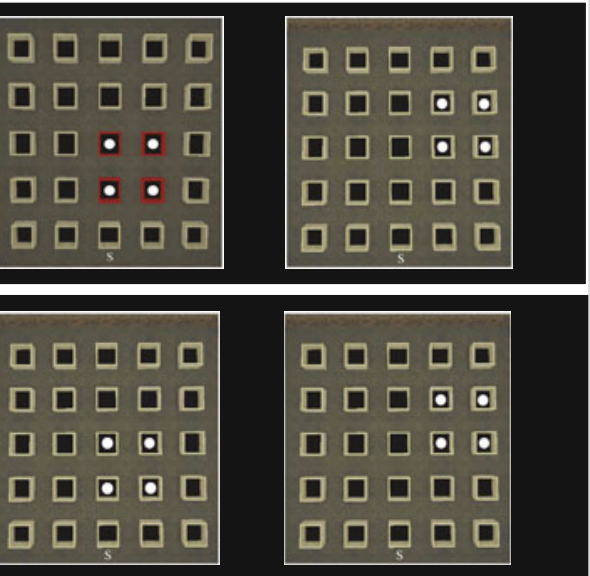

$=$ Goal location

$=$ Visual cue

$\mathrm{S}=$ Start location

Figure 1. Photos of the real environment search space (top panels) or screen shots from the firstperson perspective of the virtual environment search space (middle panels) taken at the start location (S) from two possible training trials for the cues + pattern (left) and pattern-only (right) groups. Please note that the testing trials for both groups looked identical to the pattern-only training trials (see the text for details). Bottom panel: Overhead screen shot of the virtual environment search space from two possible training trials (left and middle columns) and a testing trial (right column) for the cues + pattern (top row) and pattern-only groups (bottom row). For illustrative purposes, the white dots mark the goal locations. Red squares mark the visual cues. For illustrative purposes, the $\mathrm{S}$ marks the position where the participants entered the open field and thus started their search for all the training and testing trials. The position of the square pattern was quasirandomized across trials (see the text for details).

of the keyboard, the mouse, and the left mouse button navigated within the environment. Headphones emitted auditory feedback. Experimental events were controlled and recorded using Half-Life Dedicated Server on an identical personal computer.

\section{Stimuli}

The virtual environment $(1,050 \times 980 \times 416$ vu [length $\times$ width $\times$ height, measured in virtual units]) contained 25 raised bins $(86 \times 86 \times 38 \mathrm{vu})$ arranged in a $5 \times 5$ matrix (see Figure 1 , middle 
panels). The room was illuminated by a light source centered $64 \mathrm{vu}$ below the ceiling. The wall opposite the start location (labeled $\mathrm{S}$ in Figure 1) was lighter than the other three.

\section{Procedure}

The procedure for the virtual environment was identical to that for the real environment, with a few exceptions: (1) The participants were informed that they should locate the bins that transported them to the next virtual room, (2) the participants moved via keyboard keys ( $\uparrow$, forward; $\downarrow$, backward; $\leftarrow$, left; and $\rightarrow$, right; diagonal movement occurred if two appropriate keys were depressed simultaneously; movement of the mouse changed the view in a $360^{\circ}$ sphere within the virtual environment), (3) auditory feedback indicated movement within the environment (footstep sounds), and (4) the participants selected a bin by jumping into it. To jump into a bin, the participants simultaneously moved forward ( $\uparrow$ ) and jumped (left mouse button). Auditory feedback indicated that a jump had occurred ("huh" sound). Selection of a goal bin resulted in auditory feedback (transport sound from Super Mario Bros.). Selection of a nongoal bin resulted in different auditory feedback (game over sound from Super Mario Bros.) and required the participants to jump out of the current bin and continue searching. Successful discovery of the first, second, and third goal locations was followed by auditory feedback, but only successful discovery of all four goal locations resulted in auditory feedback and a 1-sec intertrial interval in which the monitor went black and the participants progressed to the next trial.

Training. Training in the virtual environment was identical to that in the real environment, with the exceptions that the four goal bins were marked in red for the cues + pattern group and the remaining bins were unmarked (white colored).

Testing. Testing in the virtual environment was identical to that in the real environment.

\section{RESULTS}

\section{Training}

Search behavior was influenced by the visual cues or spatial relations among goal locations. Figure 2 shows mean errors during training for locating all four goal locations (i.e., completing a trial) plotted across trial blocks for both groups and environments. A three-way mixed ANOVA on mean errors, with block (1-3), group (cues + pattern, pattern only), and environment (real, virtual) as factors, revealed main effects of block $[F(2,152)=73.83$, $p<.001]$ and group $[F(1,76)=122.49, p<.001]$ and significant block $\times$ group $[F(2,152)=15.02, p<.001]$ and group $\times$ environment $[F(1,76)=17.12, p<.001]$ interactions. No other main effects or interactions were significant $\left(F_{\mathrm{S}}<4, p \mathrm{~s}>.05\right)$. Despite differences in mean errors across environments (indicated by significant interactions), the participants in the cues + pattern group made fewer errors $(M=2.66, S E M=0.53$, and $M=0.72$, $S E M=0.26$, for real and virtual environments, respectively) than did those in the pattern-only group $(M=7.24$, $S E M=0.56$, and $M=10.76, S E M=0.75$, for real and virtual environments, respectively).

\section{Testing}

To confirm learning of the spatial relations among goal locations during testing (as opposed to a reduction in errors resulting from a decrease in return visits to any previously visited location or an increase in a tendency to select locations adjacent to a recently discovered goal location), an analysis similar to that used by Brown and Terrinoni (1996) was conducted in which the expected number of errors made to adjacent locations in the process of locating the fourth goal location, following discovery of the third goal location, was calculated.

Using the criteria that no participant made return visits to previously visited locations and that all the participants constrained their next responses to the three locations adjacent to the third goal location, three independent scenarios exist following discovery of the third goal location: (1) Two of the three adjacent locations had been previously visited, (2) one of the three adjacent locations had

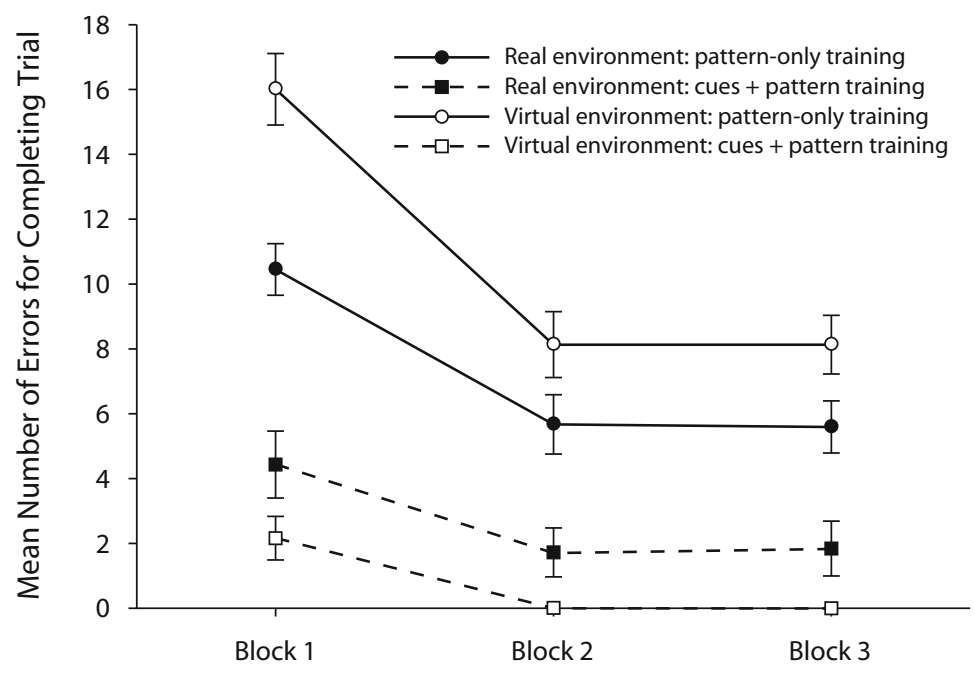

Five-Trial Blocks

Figure 2. Mean number of errors for locating all four goal locations (i.e., completing a trial) during training, plotted by five-trial blocks for each group and environment. Error bars represent standard errors of the means. 
been previously visited, and (3) none of the three adjacent locations had been previously visited.

Given that one of the three adjacent locations is the fourth goal location, each independent scenario yields an average number of errors in locating the fourth goal location following discovery of the third goal location: (1) 0 errors, (2) 0.5 errors, and (3) 1 error. Assuming equivalence in scenario likelihood, adjacent choices by participants based on chance would be expected to yield an average of 0.5 errors in the process of locating the fourth goal location after discovery of the third goal location $(0$ errors + 0.5 errors +1 error $/ 3=0.5)$. However, the participants in all the groups made significantly fewer than 0.5 errors in locating the fourth goal location following discovery of the third goal location (real environment, pattern only, $M=0.08, S E M=0.05$; real environment, cues + pattern, $M=0.09, S E M=0.09$; virtual environment, pattern only, $M=0.19, S E M=0.11$; virtual environment, cues + pattern, $M=0.03, S E M=0.02$ ), as was confirmed by one-sample, one-tailed $t$ tests $[t(19)=-7.84, p<.001$; $t(19)=-4.56, p<.001 ; t(19)=-2.82, p<.001$; and $t(19)=-28.37, p<.001$, respectively].

Although all the participants learned the spatial relations among goal locations, the participants in the cues + pattern group made fewer errors $(M=4.83, S E M=0.23)$ during testing than did those in the pattern-only group $(M=6.16, S E M=0.36)$, and despite qualitatively similar performance across environments, the participants in the real environment made fewer errors $(M=4.71, S E M=$ $0.27)$ than did those in the virtual environment $(M=6.28$, $S E M=0.32)$. A three-way mixed ANOVA on mean errors, with block (1-3), group (cues + pattern, pattern only), and environment (real, virtual) as factors, revealed main effects of block $[F(2,152)=37.55, p<.001]$, group $[F(1,76)=4.59, p<.05]$, and environment $[F(1,76)=$ $6.35, p<.05]$. No other main effects or interactions were significant $(F \mathrm{~s}<3, p \mathrm{~s}>.05)$. Figure 3 shows mean errors during testing for locating all four goal locations (i.e., completing a trial), plotted across trial blocks by group (top panel) and environment (bottom panel).

\section{DISCUSSION}

Participants in both environments learned the spatial relations among goal locations. However, during testing, the performance of the participants in both environments trained with visual cues was superior to that of those trained without these cues. Testing results indicate that the presence of the visual cues during training was not detrimental to learning the spatial relations among the goal locations. In contrast to the prediction of unitary-system models of spatial learning, no evidence was obtained for associative cue competition.

Previous failures to find cue competition in spatial learning have been accounted for by dual-system models (e.g., Burgess, 2006; Cheng, 1986; Gallistel, 1990). However, neither of these models can account for the lack of cue competition in the present experiment, because both environmental geometry and the distance of goal locations from environmental boundaries were rendered ir- relevant (e.g., goal locations varied unpredictably with respect to these cues). As a result, theoretical accounts of spatial learning that make distinctions between features and geometry (e.g., Cheng, 1986; Gallistel, 1990) or landmarks and boundaries (i.e., Burgess, 2006) must be revised to incorporate spatial relations among locations and account for their immunity to cue competition. To the extent that lack of cue competition reveals the nature of isolated modules or systems, the present results suggest that spatial relations among locations may be an important defining feature of these modules or systems used in spatial learning.

A complete account of the present results requires not only an explanation for the lack of cue competition, but also an explanation for the opposite facilitation effect of visual cue learning on learning the spatial relations among goal locations. The facilitation may be related to the weighting of information learned about the spatial relations between locations. Specifically, it has been suggested that spatial information is weighted in inverse proportion to its variance (Cheng et al., 2007). Assuming that the spatial relation between any two locations is weighted in inverse proportion to its variance and that spatial choices are based on this weighting, a goal location can serve as a useful cue for predicting the spatial location of the next goal location only if the spatial relations among goal locations are learned. As a result, discovery of each goal location would successively reduce uncertainty about the spatial location of the next goal location, but only if the spatial relations among goal locations were extracted and updated at each choice point. Importantly, uncertainty about the next goal location would decrease rapidly with successful discovery of each successive goal location.

The weightings of these spatial relations between locations would develop from experience with the consequences of spatial choices. Therefore, the participants in the pattern-only group would have weighted the spatial relations between locations solely on the basis of trial-anderror learning; however, the presence of the visual cues for participants in the cues + pattern group may have accelerated this weighting process, relative to trial-and-error learning, because the visual cues also served to constrain their spatial choices to goal locations.

An alternative explanation of facilitation may be related to information obtained through dead reckoning, a process in which an estimation of position relative to the point of departure is updated via speed of movement, direction of movement, and elapsed time of movement (for a review, see Etienne, Berlie, Georgakopoulos, \& Maurer, 1998). Specifically, it has been suggested that dead reckoning is involved not only in using spatial relations to navigate among known goal locations, but also in initial learning of the spatial relations between goal locations themselves (Brown, 2006). Movement vectors from one goal location to the next may be integrated into a representation of the spatial arrangement of goal locations. Perhaps the visual cues allowed movement vectors to be better calibrated during training for the participants in the cues + pattern group.

Although such an explanation seems problematic in accounting for the virtual environment data, since vestibular 


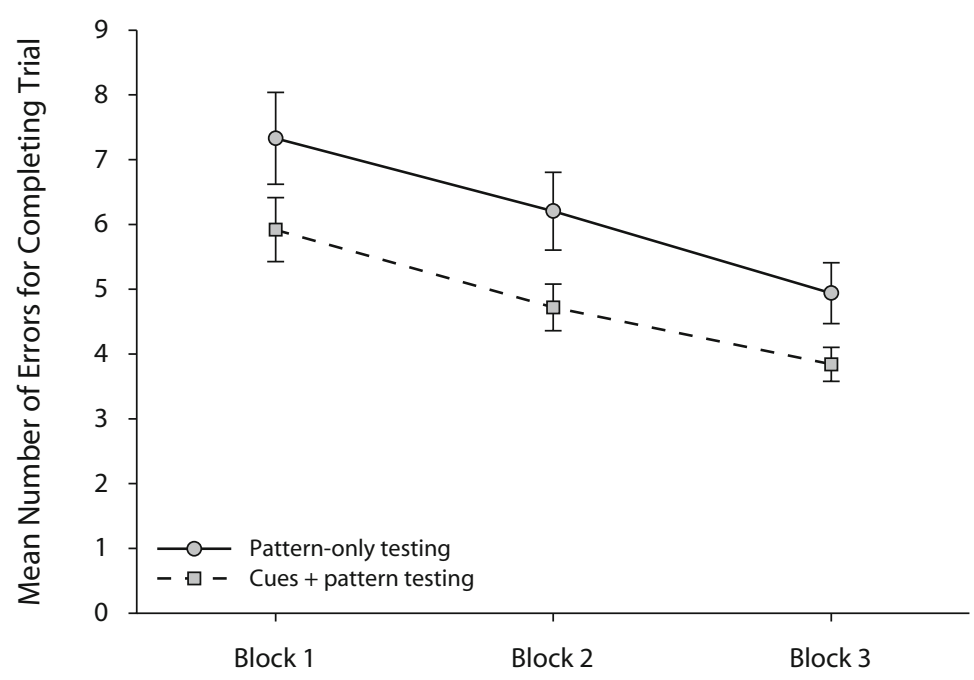

Five-Trial Blocks

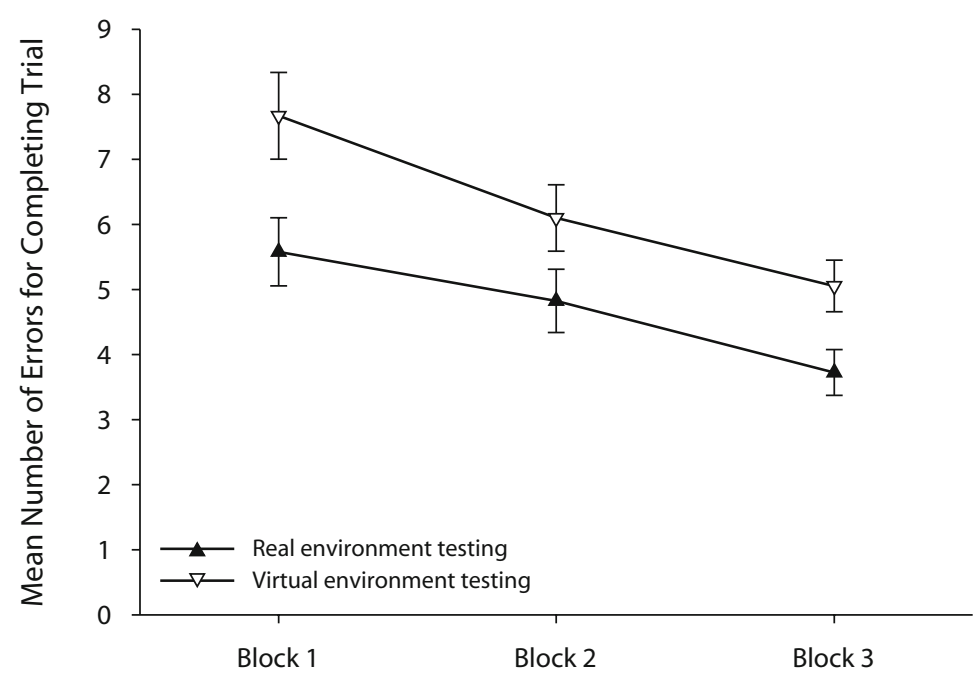

Five-Trial Blocks

Figure 3. Mean number of errors for locating all four goal locations (i.e., completing a trial) during testing plotted by five-trial blocks for group (top panel) and environment (bottom panel). Error bars represent standard errors of the means.

feedback was absent in the virtual environment task, dead reckoning has been shown to rely on both vestibular and optical information (Kearns, Warren, Duchon, \& Tarr, 2002; Nico, Israël, \& Berthoz, 2002), and investigations of dead reckoning in virtual environments have allowed researchers to eliminate vestibular information (considered the primary input for return paths in mammals) and determine that visual information - specifically, optical flow-provides sufficient information for determination of a return path (Ellmore \& McNaughton, 2004; Kearns et al., 2002). Thus, dead reckoning, based on optical flow, may be an independent mechanism for extracting spatial relations. The additional vestibular input, available only to the participants engaged in the real environment task, may have served as an additional independent mechanism in this extraction process that, coupled with the optical flow, may account for their superior performance, as compared with those engaged in the virtual environment task.

Two additional explanations for a lack of cue competition and evidence for facilitation of learning about spatial relations by visual cues should be noted: (1) verbal coding strategies employed by participants and (2) cue potentiation or feature enhancement (enhanced saliency of cues when paired together; Rescorla \& Durlach, 1981; see also Miller \& Shettleworth, 2007; Pearce et al., 2006) between the visual cues and the spatial relations. First, humans have sophisticated spatial language capacities, and it is possible that the participants used the visual cues to verbally encode the spatial relations among goal locations as an object that potentially rendered it immune to cue com- 
petition (for a review, see Plumert \& Spencer, 2007). Second, cue potentiation or feature enhancement could have occurred between the visual cues and the spatial relations among goal locations, since the visual cues were coincident with the goal locations. Although the present experiment cannot explicitly rule out these explanations, future research could address these possibilities by increasing pattern complexity and dissociating visual cues from goal locations during training.

In conclusion, the results from the present real and virtual environment search tasks appear to be inconsistent with existing dual-system accounts of spatial learning, as well as those based on standard associative accounts (for reviews, see Burgess, 2006; Chamizo, 2003; Cheng, 1986; Gallistel, 1990). Specifically, we found no evidence for cue competition in a search task when boundaries and environmental geometry were rendered irrelevant. This suggests that there are spatial cues other than environmental geometry or distance to boundaries that are immune to cue competition from landmarks. In addition, the present results add to a growing body of literature suggesting similarity in the mechanisms used by humans to navigate real and virtual environments.

\section{AUTHOR NOTE}

This research was conducted following the relevant ethical guidelines for human research and was supported by an Alzheimer Society of Canada Grant to D.M.K. We thank Chad Blair, Karen Gwillim, Jason Lukich, and Jim Reichert for their assistance with data collection and scoring. We also thank two anonymous reviewers and especially Ken Cheng for comments on an earlier version of the manuscript. Correspondence may be addressed to any author: B. R. Sturz, Department of Psychology, Armstrong Atlantic State University, 11935 Abercorn Street, Savannah, GA 31419 (e-mail: bradley.sturz@armstrong.edu); M. F. Brown, Department of Psychology, Villanova University, 800 Lancaster Avenue, Villanova, PA 19085 (e-mail: michael.brown@villanova.edu); D. M. Kelly, Department of Psychology, University of Saskatchewan, 9 Campus Drive, Saskatoon, SK, S7N 5A5 Canada (e-mail: debbie.kelly@usask.ca).

\section{REFERENCES}

BRown, M. F. (2006). Abstracting spatial relations among goal locations. In M. F. Brown \& R. G. Cook (Eds.), Animal spatial cognition: Comparative, neural, and computational approaches [Online]. Available at www.pigeon.psy.tufts.edu/asc/brown.

Brown, M. F., \& TerrinONI, M. (1996). Control of choice by the spatial configuration of goals. Journal of Experimental Psychology: Animal Behavior Processes, 22, 438-446.

Brown, M. F., YanG, S. Y., \& DiGian, K. A. (2002). No evidence for overshadowing or facilitation of spatial pattern learning by visual cues. Animal Learning \& Behavior, 30, 363-375.

Burgess, N. (2006). Spatial memory: How egocentric and allocentric combine. Trends in Cognitive Sciences, 10, 551-557.

Chamizo, V. D. (2003). Acquisition of knowledge about spatial location: Assessing the generality of the mechanism of learning. Quarterly Journal of Experimental Psychology, 56B, 102-113.

Cheng, K. (1986). A purely geometric module in the rat's spatial representation. Cognition, 23, 149-178.

Cheng, K. (2008). Whither geometry? Troubles of the geometric module. Trends in Cognitive Sciences, 12, 355-361.

Cheng, K., \& Newcombe, N. S. (2005). Is there a geometric module for spatial orientation? Squaring theory and evidence. Psychonomic Bulletin \& Review, 12, 1-23.

Cheng, K., \& Newcombe, N. S. (2006). Geometry, features, and orientation in vertebrate animals: A pictorial review. In M. F. Brown \& R. G. Cook (Eds.), Animal spatial cognition: Comparative, neural, and computational approaches [Online]. Available at www.pigeon .psy.tufts.edu/asc/cheng.

Cheng, K., Shettleworth, S. J., Huttenlocher, J., \& Rieser, J. J. (2007). Bayesian integration of spatial information. Psychological Bulletin, 133, 625-637.

Doeller, C. F., \& Burgess, N. (2008). Distinct error-correcting and incidental learning location relative to landmarks and boundaries. Proceedings of the National Academy of Sciences, 105, 5909-5914.

Doeller, C. F., King, J. A., \& Burgess, N. (2008). Parallel striatal and hippocampal systems for landmarks and boundaries in spatial memory. Proceedings of the National Academy of Sciences, 105, 5915-5920.

Ellmore, T. M., \& McNaughton, B. L. (2004). Human path integration by optic flow. Spatial Cognition \& Computation, 4, 255-272.

Etienne, A. S., Berlie, J., Georgakopoulos, J., \& Maurer, R. (1998). Role of dead reckoning in navigation. In S. Healy (Ed.), Spatial representation in animals (pp. 54-68). New York: Oxford University Press.

Gallistel, C. R. (1990). The organization of learning. Cambridge, MA: MIT Press.

Graham, M., Good, M. A., McGregor, A., \& Pearce, J. M. (2006). Spatial learning based on the shape of the environment is influenced by properties of the objects forming the shape. Journal of Experimental Psychology: Animal Behavior Processes, 32, 44-59.

Healy, S. (1998). Spatial representation in animals. New York: Oxford University Press.

Kearns, M. J., Warren, W. H., Duchon, A. P., \& Tarr, M. (2002). Path integration from optic flow and body senses in a homing task. Perception, 31, 349-374.

Kelly, D. M., \& Gibson, B. M. (2007). Spatial navigation: Spatial learning in real and virtual environments. Comparative Cognition \& Behavior Reviews, 2, 111-124.

Klatzky, R. L., Loomis, J. M., Beall, A. C., Chance, S. S., \& Golledge, R. G. (1998). Spatial updating of self-position and orientation during real, imagined, and virtual locomotion. Psychological Science, 9, 293-298.

Miller, N. Y., \& Shettleworth, S. J. (2007). Learning about environmental geometry: An associative model. Journal of Experimental Psychology: Animal Behavior Processes, 33, 191-212.

Nico, D., IsRaËL, I., \& Berthoz, A. (2002). Interaction of visual and ideothetic information in a path completion task. Experimental Brain Research, 146, 379-382.

Pearce, J. M., Graham, M., Good, M. A., Jones, P. M., \& McGregor, A. (2006). Potentiation, overshadowing, and blocking of spatial learning based on the shape of the environment. Journal of Experimental Psychology: Animal Behavior Processes, 32, 201-214.

Plumert, J. M., \& SPencer, J. P. (2007). The emerging spatial mind. New York: Oxford University Press.

Rescorla, R. A., \& Durlach, P. (1981). Within-event learning in Pavlovian conditioning. In N. E. Spear \& R. R. Miller (Eds.), Information processing in animals: Memory mechanisms (pp. 81-111). Hillsdale, NJ: Erlbaum.

Shettleworth, S. J. (1998). Cognition, evolution, and behavior. New York: Oxford University Press.

Sturz, B. R., Bodily, K. D., Katz, J. S., \& Kelly, D. M. (2009). Evidence against integration of spatial maps in humans: Generality across real and virtual environments. Animal Cognition, 12, 237-247. doi:10.1007/s10071-008-0182-z

(Manuscript received May 5, 2008; revision accepted for publication September 24, 2008.) 